\title{
Estado nutricional e percepção da imagem corporal de mulheres idosas residentes no nordeste do Brasil
}

\author{
Nutritional status and perceived body image \\ of elderly women in the Northeast of Brazil
}

Sheila Tribess $^{1}$
Jair Sindra Virtuoso Junior ${ }^{1}$
Édio Luiz Petroski $^{2}$

${ }^{1}$ Departamento deEducação Física, Universidade Federal do Triângulo M ineiro. Av. Frei Paulino, 30, Abadia. 64049-550 Uberaba M G. sheillatribess@yahoo.com.br

${ }^{2}$ Programa de PósGraduação em Educação

Física, Centro de Desportos, UniversidadeFederal de Santa Catarina.

Abstract This paper aims to investigatetheassociation between perceived body image and nutritional status in elderly women. The methodology used was a cross-sectional cohort study of a sample of 265 elderly women aged 60 to 96 and selected by randomized proportional stratification by groups of convenience in the town of Jequié, Bahia, Brazil. Each subject was interviewed in order to obtain information on sociodemographic characteristics (age, marital status, educational level and economic class) and perceived body image (on a nine-silhouette scale) and their body mass and height weremeasured in order to calculatetheir Body M ass Index as a parameter of nutritional status. Data analysis employed descriptive statistics, measures of association and nonparametric analysis. A total of $43.8 \%$ of theelderly women interviewed were well-nourished, 3.4\% were underweight and $52.8 \%$ were overweight. With reference to perceived body image, $54 \%$ of the women were unsatisfied, primarily due to excess body mass (35.1\%), and this was associated with nutritional status, in that increases in BM I raised the percentage of unsatisfied women. The elderly from Bahia were unsatisfied with their body images, and this dissatisfaction was associated with the prevalence of obesity. Key words Body image, Nutritional status, Elderly, Women
Resumo 0 objetivo deste estudo foi identificar a associação da percepção da imagem corporal com o estado nutricional de mulheres idosas. Trata-se deum estudo transversal com 265 idosas, na faixa etária de 60 a 96 anos, selecionada deforma aleatória e estratificada proporcional por grupos de conveniência no município de Jequié, Bahia. Foi aplicada uma entrevista com o intuito de obter informações referentes às características sociodemográficas (idade, estado civil, escolaridadeeclasse econômica), percepção da imagem corporal (escala de nove silhuetas) e mensuração da massa corporal eestatura para cál culo do I I ndice de M assa Corporal como parâmetro do estado nutricional. Utilizaram-se os procedimentos da estatística descritiva, medidas de associação e análise não paramétricas. Das idosas entrevistadas, 43,8\% eram eutróficas, 3,4\% se encontravam na categoria de baixo peso e $52,8 \%$ apresentavam sobrepeso. Q uanto à percepção da imagem corporal, 54\% das idosas eram insatisfeitas, principalmente pelo excesso corporal ( $35,1 \%)$, que estava associado ao estado nutricional, no qual o aumento da categoria do IM C elevava o percentual deidosas insatisfeitas. As idosas estavam insatisfeitas com sua imagem corporal eessa insatisfação estava associada à prevalência de obesidade.

Palavras-chave Imagem corporal, Estado nutricional, Idoso, Mulher 
Introdução

As mudanças ocorridas em determinados componentes da composição corporal, a exemplo da diminuição do tecido adiposo e do aumento de massa muscular, decorrentes do exercício físico e da dieta alimentar, são responsáveis pela maior associação entre a percepção da imagem corporal e os valores reais das variáveis antropométricas, o que leva as pessoas a uma sensação de bem-estar ${ }^{1}$

Dentre os principais motivos que levam os idosos a procurarem práticas de atividades físicas, estão os benefícios proporcionados à saúde, quais sejam: 0 sentir-se bem fisicamente ${ }^{2}$, controle de peso corporal, melhora da aparência ${ }^{2} \mathrm{e}$ da aptidão física ${ }^{3}$ e redução do estresse $e^{4}$.

A procura por um peso corporal reduzido, visando a uma aparência corporal aceita pelos padrões de magreza, impostos pela sociedade atual, pode estar associada a hábitos alimentares inadequados, queseiniciam na adolescência epersistem até a senilidade ${ }^{5,6}$. A dieta com restrição calórica tem sido reportada como o método mais comumente utilizado pelas mulheres para a perda de peso, em busca de uma satisfação corporal ${ }^{3,7,8}$.

A insatisfação com a forma corporal e a fixação pela magreza fazem com que mulheres restrinjam, em sua dieta, alimentos nutritivos ne cessários para o adequado funcionamento do organismo, ocasionando sérios problemas comportamentais e de saúde?.

A avaliação da percepção da aparência corporal por meio da escala de silhueta corporal está relacionada com o indicador do índice de massa, uma vez que possui grande variabilidade para classificar o indivíduo em baixo peso, normal, sobrepeso e obesidade ${ }^{10,11}$.

0 propósito deste estudo foi analisar a relação entre a percepção da imagem corporal com o estado nutricional de mulheresidosas residentes no nordeste do Brasil. Tais informações possibilitarão a ampliação do conhecimento sobreo comportamento das variáveis investigadas em pessoas idosas, bem como fundamentar a elaboração de políticas públicas direcionadas ao controle e/ou redução do sobrepeso/obesidade em pessoas idosas.

\section{Métodos}

A população desteestudo compreen deu quinhentas pessoas do sexo feminino, entre 60 e 95 anos de idade, inscritas em grupos de convivência do município de Jequié, Bahia, região nordeste do Brasil. A amostra do tipo probabilística estratificada proporcional por grupo de convivência foi selecionada de forma aleatória, sendo composta por 265 idosas, estabelecendo-senível deconfiança de $95 \%$, com erro de $5 \%$.

Para a avaliação, foi elaborada uma entrevista multidimensional que foi aplicada de forma individual, contendo informações sociodemográficas referentes à idade, escolaridade, estado civil, classe econômica, situação ocupacional, arranjo familiar e condições de saúde autoreferida.

0 estado nutricional foi analisado pelo Índice de M assa Corporal (IMC), que utiliza a relação massa corporal $(\mathrm{kg})$, dividida pela estatura (m) ao quadrado. Para isso, foram mensuradas as variáveis antropométricas, massa corporal e estatura, com o auxílio de uma balança digital de marca Filizola e um estadiômetro, adotando-se os procedimentos padronizados ${ }^{12}$. A classificação do IM C obedeceu os critérios da Organização M undial da Saúde ${ }^{13}$, que propõem a classificação em peso baixo $\left(<18,5 \mathrm{Kg} \cdot \mathrm{m}^{-2}\right)$, normal $\left(18,5-24,9 \mathrm{Kg}^{-\mathrm{m}^{-2}}\right.$ ) e sobrepeso (e $\left.\geq 25 \mathrm{Kg} \cdot \mathrm{m}^{-2}\right)$.

A imagem corporal foi verificada pela escala denove silhuetas, proposta por Stunkard et al. ${ }^{14}$, que representa um continuum desde a magreza (silhueta 1 ) até a obesidade severa (silhueta 9), na qual a idosa escolhe o número da silhueta que considera mais semelhante a sua aparência corporal real e também o número da silhueta que acredita ser a aparência corporal ideal, considerada para sua idade.

Para avaliação da imagem corporal, subtraiuse a aparência corporal real da aparência corporal ideal. Quando a variação era igual a zero, classificava-se a idosa como satisfeita; e se diferente de zero, classificava-se como insatisfeita. Caso a diferença fosse positiva, era uma insatisfação pelo excesso de peso; e, quando negativa, uma insatisfação pela magreza.

Para confecção do banco de dados, foi utilizado o programa Epidata, versão 2.1b, e as análises foram feitas por meio do software SPSS, versão 11.0. A análise descritiva dos dados serviu para caracterizar a amostra, com a distribuição da frequência, cálculo de tendência central (mé dia e mediana) e de dispersão (amplitude de variação, desvio padrão e intervalo de confiança).

A normalidade dos dados foi verificada pelo teste de Kolmogorov-Smirnov para todas as variáveis analisadas. Para as variáveis quenão apresentaram uma distribuição normal, foram utilizados testes não-paramétricos. Os testes utilizados foram o qui-quadrado para verificar a asso- 
ciação entre as variáveis categóricas, percepção da imagem corporal eestado nutricional; o $U$ de $M$ ann-Whitney para verificar as diferenças da percepção da imagem corporal (satisfeito einsatisfeito) em relação ao estado nutricional; 0 Kruskal-Wallis para verificar as diferenças do valor do IMC em relação à faixa etária e percepção da imagem corporal (satisfeito, insatisfeito pela magreza e insatisfeito pelo excesso). Para todos os procedimentos de análise, foi adotado o nível de significância de $5 \%(p<0,05)$.

Esta pesquisa seguiu os princípios éticos presentes na Declaração de H elsinque e na Resolução no 196/96 do Conselho N acional de Saúde. Os protocolos de pesquisa foram avaliados e aprovados pelo ComitêdeÉtica em Pesquisa com Seres Humanos da Universidade Federal de Santa Catarina (Parecer no 049/2005).

Tabela 1. Características sociodemográficas da amostra.

\begin{tabular}{|c|c|c|}
\hline Variável & $\%$ & $\mathrm{n}$ \\
\hline \multicolumn{3}{|l|}{ Faixa etária } \\
\hline 60-64 anos & 17,7 & 47 \\
\hline 65-69 anos & 29,8 & 79 \\
\hline 70-74 anos & 24,5 & 65 \\
\hline 75-79 anos & 14,0 & 37 \\
\hline 80-84 anos & 8,3 & 22 \\
\hline Acima de 85 anos & 5,7 & 15 \\
\hline \multicolumn{3}{|l|}{ Estado civil } \\
\hline Solteira & 11,3 & 30 \\
\hline Casada/vivendo com o parceiro & 28,3 & 75 \\
\hline Viúva & 48,3 & 128 \\
\hline $\begin{array}{l}\text { Divorciada ou separada/ } \\
\text { desquitada }\end{array}$ & 12,1 & 32 \\
\hline \multicolumn{3}{|l|}{ Escolaridade } \\
\hline Fundamental incompleto & 88,7 & 235 \\
\hline $\begin{array}{l}\text { Fundamental completo/ médio } \\
\text { incompleto }\end{array}$ & 3,0 & 08 \\
\hline $\begin{array}{l}\text { M édio completo/superior } \\
\text { incompleto }\end{array}$ & 7,2 & 19 \\
\hline Superior completo & 1,1 & 03 \\
\hline \multicolumn{3}{|l|}{ O cupação } \\
\hline Aposentada, mas trabalha & 4,2 & 11 \\
\hline Só aposentada & 58,5 & 155 \\
\hline Só dona de casa & 15,1 & 40 \\
\hline Pensionista & 22,3 & 59 \\
\hline \multicolumn{3}{|l|}{ Classe econômica (ANEP) } \\
\hline B1 & 1,9 & 05 \\
\hline $\mathrm{B}_{2}$ & 6,4 & 17 \\
\hline$C^{2}$ & 22,6 & 60 \\
\hline$D$ & 55,8 & 148 \\
\hline$E$ & 13,2 & 35 \\
\hline
\end{tabular}

\section{Resultados}

As características sociodemográficas dos participantes do estudo $(n=265)$ são apresentadas na Tabela 1 e compreendem idade, estado civil, escolaridade, situação ocupacional e nível socioeconômico.

A média de idade das mulheres foi de 71,15 anos ( $D P=7,44)$, com idade variando de 60 a 96 anos, com predominância de viúvas, aposentadas, pertencenteà classeeconômica $\mathrm{D}$ e com baixo nível de escolaridade (fundamental incompleto).

A classificação das idosas de acordo com o IMC, utilizada para analisar o estado nutricional, é apresentada na Figura 1. A média do IM C das idosas foi $25,84 \mathrm{~kg} \cdot \mathrm{m}^{-2}(\mathrm{DP}=4,56)$, com amplitude de 15,64 a $39,52 \mathrm{~kg} \cdot \mathrm{m}^{-2}$. Das idosas avaliadas, 43,8\% eram eutróficas; 3,\% foram classificadas na categoria de baixo peso e $52,9 \%$, na categoria de sobrepeso.

$\mathrm{Na}$ análise da percepção da imagem corporal, $46 \%(n=122)$ das idosas estavam satisfeitas com sua imagem corporal, pois escolheram a siIhueta real igual à ideal, e 54\% (143), insatisfeitas. Das idosas insatisfeitas, 34,9\% ( $n=50)$ estavam insatisfeitas com a "magreza", e 65,1\% (n=93) insatisfeitas com o excesso de peso corporal (Figura 2).

Quando comparada às categorias da imagem corporal com a idade, massa corporal, estatura e IM C, observou-se que apenas a massa corporal eo IM C apresentaram diferenças significativas (Tabela 2).

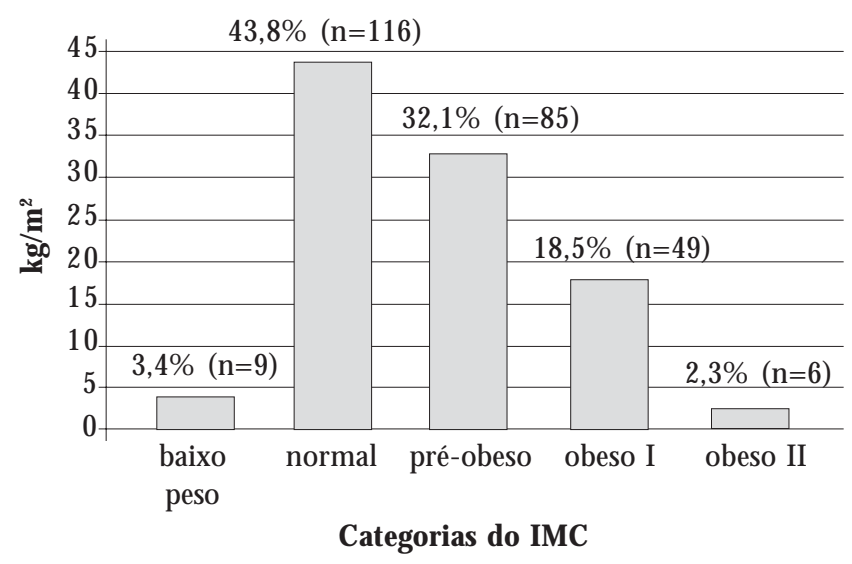

Figura 1. Classificação das idosas de acordo com o índice de massa corporal. 


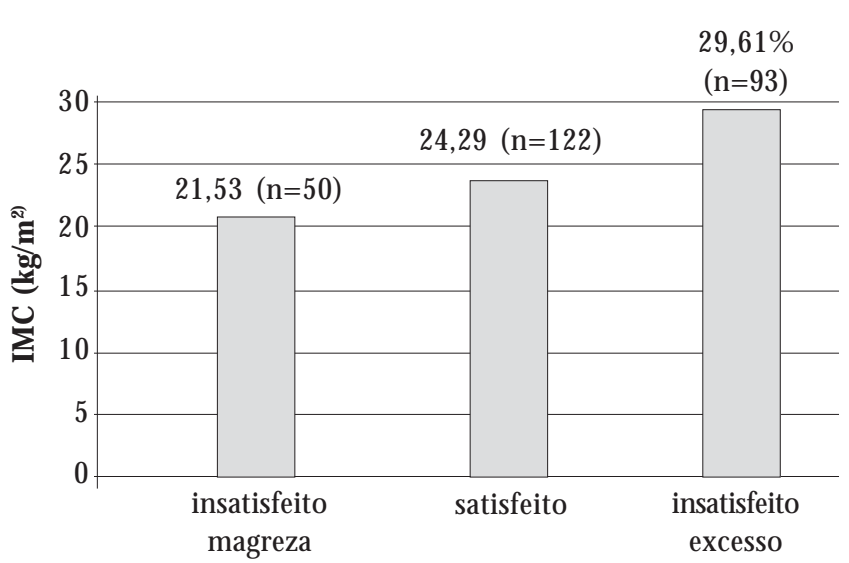

Percepção da imagem corporal

Figura 2. Distribuição do IMC das idosas em relação à percepção da imagem corporal (satisfeito, insatisfeito pela magreza e insatisfeito pelo excesso)

Tabela 2. M ediana e rank médio da idade, massa corporal, estatura e I M C das idosas de acordo com a percepção da imagem corporal.

\begin{tabular}{lrrcc}
\hline \multicolumn{1}{c}{ Variáveis } & Mediana & $\begin{array}{c}\text { Rank } \\
\text { médio }\end{array}$ & U & $p$ \\
\hline Idade (anos) & & & & \\
$\quad$ Satisfeita & 70,5 & 137,12 & 8220,5 & 0,419 \\
Insatisfeita & 70,0 & 129,49 & & \\
$\quad$ Total & 70,0 & & & \\
Massa corporal (kg) & & & & \\
$\quad$ Satisfeita & 53,7 & 112,27 & 6194,0 & 0,000 \\
Insatisfeita & 61,7 & 150,69 & & \\
$\quad$ Total & 57,5 & & & \\
Estatura (anos) & & & & \\
$\quad$ Satisfeita & 150,0 & 127,25 & 8021,5 & 0,259 \\
Insatisfeita & 150,5 & 137,91 & & \\
$\quad$ Total & 150,5 & & & \\
IM C (Kg.m-2) & & & & \\
Satisfeita & 24,29 & 111,66 & 6119,0 & 0,000 \\
Insatisfeita & 27,77 & 151,21 & & \\
$\quad$ Total & 25,31 & & & \\
\hline
\end{tabular}

A mediana de idade das idosas satisfeitas com a imagem corporal não diferiu significativamente $(U=8220,5$ e $p=0,419)$ das idosas insatisfeitas, mesmo quando analisada a percepção da imagem corporal em três categorias: satisfeita, insatisfeita pela magreza e insatisfeita pelo excesso $\left(\chi^{2}=5,87\right.$ e $\left.p=0,53\right)$. Assim como para a estatura das idosas satisfeitas e insatisfeitas com a imagem corporal $(U=8021,5$ e $p=0,259)$.
Já a mediana da massa corporal das idosas foi $57,5 \mathrm{~kg}$, sendo que as mulheres insatisfeitas com a imagem corporal apresentaram maior massa corporal $(61,70 \mathrm{~kg})$ quando comparadas às idosas satisfeitas com a imagem corporal $(53,70 \mathrm{~kg})$, com diferença estatisticamente significativa $(p=0,000)$.

Quando analisado o IMC das idosas em relação à percepção da imagem corporal em duas categorias (satisfeito e insatisfeito), verificou-se diferença significativa no valor do IMC $(\mathrm{U}=6119,0, \mathrm{p}=000)$. As idosas satisfeitas apresentaram um IMC de $24,29 \mathrm{~kg} \cdot \mathrm{m}^{-2}$, classificado como normal, enquanto que as idosas insatisfeitas apresentaram valor superior para o IMC, 27,77 kg.m-2 , classificado como sobrepeso.

A análise do teste de Kruskal-Wallis revelou diferença significativa na distribuição do IM C em relação à percepção da imagem corporal em três categorias: satisfeito, insatisfeito pela magreza e insatisfeito pelo excesso $\left(\chi^{2}=91,57\right.$ e $\left.p=0,000\right)$. $\mathrm{Na}$ Figura 2, pode-se constatar o aumento significativo do valor da mediana do IM C entre as categorias do insatisfeito pela magreza, satisfeito e insatisfeito pelo excesso.

Quando efetuado o teste de qui-quadrado entre as categorias do IM C com a percepção da imagem corporal (satisfeito e insatisfeito), verificou-se associação significativa $\left(\chi^{2}=25,52\right.$ e $p=0,000$ ) (Tabela 3).

Observa-se uma maior proporção de idosas insatisfeitas com a imagem corporal nas categorias de baixo peso $(88,9 \%, n=8)$ e sobrepeso $(65,7 \%, n=92)$, o que não acontece na categoria

Tabela 3. Frequência absoluta e relativa das idosas segundo as categorias do I M C e percepção da imagem corporal.

\begin{tabular}{|c|c|c|c|c|c|c|}
\hline \multirow{3}{*}{$\begin{array}{c}\text { Categorias } \\
\text { do IMC }\end{array}$} & \multicolumn{6}{|c|}{ Percepção da imagem corporal } \\
\hline & \multicolumn{2}{|c|}{ satisfeita } & \multicolumn{2}{|c|}{ insatisfeita } & \multirow[b]{2}{*}{$x^{2}$} & \multirow[b]{2}{*}{$p$} \\
\hline & $\mathrm{n}$ & $\%$ & $n$ & $\%$ & & \\
\hline Baixo & 01 & 11,1 & 08 & 88,9 & 25,52 & $0,000^{\mathrm{a}}$ \\
\hline Normal & 73 & 62,9 & 43 & 37,1 & 8,43 & $0,004^{b}$ \\
\hline Sobrepeso & 48 & 34,3 & 92 & 65,7 & & \\
\hline Préobeso & 39 & 45,9 & 46 & 54,1 & & \\
\hline Obeso I & 09 & 18,4 & 40 & 81,6 & 37,96 & $0,000^{\mathrm{a}}$ \\
\hline Obeso II & - & - & 06 & 100 & 20,53 & $0,000^{b}$ \\
\hline
\end{tabular}

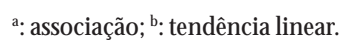


normal, em que a maioria $(62,9 \%, n=73)$ das idosas está satisfeita com sua imagem corporal.

A associação para tendência linear ficou mais evidente quando se dividiu a categoria de sobrepeso em pré-obeso, obeso I eobeso II. Observouse que, quanto mais elevada a categoria de IM C, maior era o percentual de idosas insatisfeitas com imagem corporal em comparação ao percentual de idosas satisfeitas, sendo que, na categoria de obeso I, 81,6\% das idosas estavam insatisfeitas, e na categoria obeso II, 100\% das idosas estavam insatisfeitas com a imagem corporal.

\section{Discussão}

0 presenteestudo levou em conta a heterogeneidade dos treze grupos de convivência, vinculados à Associação de Amigos, Grupos de Convivência e Universidade Aberta a Terceira Idade (AAGRUTI), localizados em oito bairros do município de Jequié (BA); procurou-se analisar a percepção da imagem corporal em diferentes estratos socioeconômicos.

0 presente estudo constituiu-se de mulheres idosas com faixa etária condizentes com a realidade brasileira de acordo com os Indicadores Sociais do IBGE ${ }^{15}$, nos quais $50 \%$ dosidosos brasileiros estão na faixa etária de 60 a 69 anos e os outros $50 \%$ são representados por idosos acima de 70 anos. No presente estudo, $47,5 \%$ das muIheres pertenciam à faixa etária de 60 a 69 anose $52,5 \%$ eram maiores que 70 anos; destaca-seainda que $14 \%$ das idosas têm mais de 80 anos.

Quando analisado o nível de escolaridade das idosas, observou-se um baixo índice, em que $88,7 \%(n=235)$ das idosas não completaram o ensino fundamental, similares a outros estudos ${ }^{15-}$ 17; $(n=8)$ possuíam o ensino fundamental completo; $7,2 \%(n=19)$ das idosas cursaram o ensino médio completo, eapenas $1,1 \%(n=3)$ das idosas possuíam o ensino superior completo. Observase grande contraste educacional quando comparado a idosos de Florianópolis, onde aproximadamente $12 \%$ possuem o nível superior ${ }^{18}$.

0 nordeste é uma região economicamente pobre do país, verificado pelos dados da Pesquisa Nacional por Amostra de Domicílios $(P N A D)^{15}$, a qual constatou que os residentes na zona rural e os residentes nas regiões norteenordeste possuem a menor renda domiciliar per capita. Esses resultados são confirmados pelo baixo nível econômico das idosas, a maioria ( $69 \%$ e $\mathrm{n}=183$ ) pertence às classes $\mathrm{D}$ eE e sobrevive com atéum salário mínimo (51,7\% en=137). 0 nível econômico das idosas demonstrou características similares à distribuição econômica da população nordestina ${ }^{15}$.

0 nordeste, assim como outras regiões pobres do país, vem passando por um fenômeno denominado de transição nutricional, em que 0 problema de escassez alimentar tem sido substituído pelo excesso, quevem gerando um aumento de sobrepeso e obesidade ${ }^{19}$. 0 expressivo aumento do sobrepeso na população idosa brasileirajáéevidenciado com maisintensidade, principalmente nas classes econômicas intermediárias e baixas ${ }^{20}$. Esse fenômeno pode ser constatado com as idosas do presente estudo, das quais $52,8 \%$ apresentavam sobrepeso.

0 sobrepeso e a obesidade são fatores de riscos à saúde. Entre os mais frequentes agravos ocasionados pelo sobrepeso e obesidade estão as doenças isquêmicas do coração, hipertensão arterial, acidente vascular cerebral, diabetes mellitus tipo 2, osteoartrite, neoplasias, esofagite de refluxo, problemas psicológicos, entreoutros ${ }^{13,21}$.

Por outro lado, o baixo peso apresentado em $3,4 \%(n=9)$ das idosas é merecedor de atenção, devido a sua associação com estado de desnutrição, predispondo a uma série de complicações graves à saúde, incluindo tendência à infecção, deficiência de cicatrização deferidas, falência respiratória, insuficiência cardíaca, diminuição da síntese de proteínas a nível hepático com produção de metabólicos anormais, diminuição da filtração glomerular e da produção de suco gástri$\mathrm{CO}^{22}$. As condições de magreza excessivas estão mais fortementeassociadas à mortalidade do que 0 excesso de peso ${ }^{23,24}$.

Em relação à percepção da imagem corporal, evidenciaram-se diferenças na escolha das silhuetas real e ideal, que gerou a insatisfação com a aparência corporal em $54 \%(n=143)$ dasidosas e principalmente pelo excesso de peso corporal.

Resultados com maior prevalência de insatisfação foram evidenciados em uma pesquisa ${ }^{25}$, utilizando o mesmo instrumento de medida do presente estudo, com mulheres de 50 a 80 anos, de classe econômica média a baixa, com mais de cinco anos de escolaridade, que frequentavam as aulas de ginástica do projeto Longitudinal de Aptidão Física e Envelhecimento de São Caetano do Sul. No referido estudo, foi constatado que apenas $28,2 \%$ das mulheres estavam satisfeitas com a aparência corporal, e 71,7\%, insatisfeitas, sendo que $67,4 \%$ das mulheres desejavam possuir uma aparência mais magra e 4,3 \% das muIheres, uma aparência mais gorda. Entretanto, ressalta-se que as idosas apresentavam um alto 
IM C; $75 \%$ apresentavam sobrepeso (eH 25 kg.m

$\left.{ }^{2}\right)$, valor superior ao presente estudo, no qual $52,8 \%$ das idosas tinham sobrepeso.

0 entendimento dos aspectos que influenciam a satisfação da imagem corporal no idoso é determinante na elaboração de intervenções direcionadas ao bem-estar do mesmo. Algumas evidências na literatura demonstram que há um incremento da insatisfação corporal com o aumento dos níveis de sobrepeso eobesidade, principalmente na mulher ${ }^{9,22-28 .}$

Esses resultados foram evidenciados no estudo em questão, no qual se constatou que valores extremos, tanto os mais baixos como os elevados para o IMC, ocasionavam uma maior insatisfação com a imagem corporal. Das idosas queapre sentavam sobrepeso, 65,7\% ( $n=92$ ) estavam insatisfeitas com sua imagem corporal e, à medida que aumentava o valor do IM C, essa insatisfação também aumentava significativamente.

Alguns estudos indicam que o IM C em muIheres está inversamente relacionado com a satisfação com o peso corporal ${ }^{6,29,30}$, mesmo quando as análises eram estratificadas pelo nível de atividadefísica ${ }^{29}$. Tais informações são semelhantes aos dados encontrados na amostra deste estudo, que apresentaram maior insatisfação nos grupos de IMC mais elevado, independente do nível de atividade física habitual. Há relatos na literatura que mulheres com peso adequado mostravam-se insatisfeitas com a com sua imagem corporal ${ }^{30}$.

A auto-avaliação da percepção da imagem corporal parece servir como instrumento capaz de estimar com razoável precisão o estado nutricional de mulheres idosas. No entanto, fatores relacionados à saúde, a exemplo da presença de condiçõesfísicas (doenças), o baixo nível socioeconômico e de escolaridade podem ter limitado a percepção mais apurada da imagem corporal.

\section{Conclusão}

A prevalência de sobrepeso atinge metade da população. A insatisfação com a forma corporal predomina entre as idosas. 0 estudo evidenciou a associação entre sobrepeso e insatisfação corporal.

Os resultados apresentados evidenciam a necessidade de programas direcionados ao controle do peso corporal, em busca de um melhor estado nutricional que minimizea insatisfação corporal, bem como neutralizea pressão social exercida na busca as formas corporais mais magras.

\section{Colaboradores}

STribess JSVirtuoso Junior participaram da elaboração do artigo nas fases de concepção, delineamento, análise e interpretação dos dados e redação do artigo. EL Petroski participou das fases de análise e interpretação dos dados e revisão crítica da redação final. 
1. Boutcher S. Emotions and aerobic exercise. In: Singer R, Murphey M, Ternnant $L$, editors. Handbook of research on sport psychology. New York: Macmillan Publishing; 1993. p. 799-914.

2. Schuler PB, Broxon-Hutcherson A, Philipp SF, Ryan S, Isosaari RM, Robinson D. Body-shape perceptions in older adults and motivations for exercise. Percept M ot Skill. 2004; 98(3 Pt 2):1251-1260.

3. Araújo DS, Araújo CG. Self-perception and dissatisfaction with weight does not depend on the frequency of physical activity. Arq Bras Cardiol 2003; 80(3):235-249.

4. Rumsey N, Clarke A, White P, Wyn-Williams M, Garlick W. Altered body image: appearance-related concerns of people with visible disfigurement. J Adv Nurs 2004; 48(5):443-453.

5. Eiting S, Platen P. Eating behavior and body perception in female participants of fitness courses in sport studios. M ed Sci Sports Exerc 2002; 34(5 suppl):S172.

6. Stevens J, Kumanyika SK, Keil JE. Attitudes toward body size and dieting: differences between elderly black and white women. Am J Public Health 1994; 84(8):1322-1325.

7. Anderson LA, Eyler AA, Galuska DA, Brown DR, Brownson RC. Relationship of satisfaction with body size and trying to lose weight in a national survey of overweight and obese women aged 40 and older, United States. Prev Med 2002; 35(4):390-396.

8. O'Brien K, Venn BJ, Perry T, Green TJ, Aitken AB, Thomson R. Reasons for wanting to lose weight: different strokes for different folks. Eat Behav 2007;(1):132-135.

9. Hrabosky JI, Grilo CM. Body image and eating disordered behavior in a community sample of Black and Hispanic women. Eat Behav 2007; 8(1):106-114.

10. Mueller WH, Joos SK, Schull WJ. Alternative measurements of obesity: accuracy of body silhouettes and reported weights and heights in a Mexican American sample. Int J Obes 1985; 9(3):193-200.

11. Tehard B, Van Liere MJ, Com Nougue C, ClavelChapelon F. Anthropometric measurements and body silhouette of women: validity and perception. J Am Diet Assoc 2002; 102(12):1779-1784.

12. Alvarez BR, Pavan AL. Alturas e Comprimentos. In: Petroski EL, organizador. Antropometria: técnicas e padronizações. 2 ${ }^{\mathrm{a}}$ ed. Porto Alegre: Pallotti; 2003. p. 31-47.

13. World Health Organization. O besity: preventing and managing the global epidemic. Report of a WHO consultation on obesity. Geneva: WH O; 1998.

14. Stunkard AJ, Sorensen T, Schlusinger F. Use of the Danish Adoption Register for the study of obesity and thinness. In: Kety SS, Rowland LP, Sidman RL, $M$ atthysse SW, editors. Genetics of neurologic and psychiatric disorders. New York: Raven Press; 1983. p.115-120.

15. Instituto Brasileiro de Geografia e Estatística. Estudos e pesquisas informação demográfica - Síntese de indicadores sociais, 2004. Rio de Janeiro: IBGE; 2005.
16. Lebrão ML. O projeto SABE em São Paulo: uma visão panorâmica. In: Lebrão $M L$, Duarte YAO, organizadores. SABE - Saúde, Bem-estar e Envelhecimento - O projeto Sabe no município de São Paulo: uma abordagem inicial. Brasília: Organização PanAmericana da Saúde; 2003.

17. Barreto KML, Carvalho EM F, Falcão IV, Lessa FJD, Leite VMM . Perfil sócio-epidemiológico demográfico das mulheres idosas da Universidade Aberta à Terceira Idade no estado de Pernambuco. Rev Bras Saude M ater. Infant 2003; 3(3):339-354.

18. Benedetti TRB, Melo ALSF, Gonçalves LT. Idosos de Florianópolis: autopercepção das condições de saúde bucal e utilização de serviços odontológicos. Cien Saude Colet 2007; 12(6):1683-1690.

19. Coitinho DC, Leão $M M$, Recine $E$, Sichieri R. Condições nutricionais da população brasileira: adultos e idosos. Pesquisa nacional sobre saúde e nutrição. Brasília: INAN; 1991.

20. Tavares EL, Anjos LA. Perfil antropométrico da população idosa brasileira: Resultados da Pesquisa N acional de Saúde e Nutrição. Cad Saude Publica 1999; 15(4):759-768.

21. Intorre $F, M$ aiani G, Cuzzolaro M, Simpson EE, Catasta G, Ciarapica D, Mauro B, Toti E, Zaccaria M, Coudray C, Corelli S, Palomba L, Polito A. Descriptive data on lifestyle, anthropometric status and mental health in Italian elderly people. J N utr Health Aging 2007; 11(2):165-174.

22. Organização Mundial da Saúde. Manejo da desnutrição grave: um manual para profissionais de saúde de nível superior e suas equipes auxiliares. Genebra: OM S; 2000.

23. Landi F, Zuccala G, Gambassi G, Incalzi RA, Manigrasso L, Pagano F, Carbonin P, Bernabel R. Body mass index and mortality among older people living in the community. J Am Geriatr Soc 1999; 47:10721076.

24. Otero UB, Rozenfeld S, Gadelha AMJ, Carvalho MS. M ortalidade por desnutrição em idosos, região sudeste do Brasil, 1980-1997. Rev. Saude Publica 2002; 36(2):141-148.

25. Braggion GF. Satisfação com a aparência corporal, nível de atividade física, valor calórico da dieta e estado nutricional de mulheres com 50 anos e mais de acordo com o grupo etário [dissertação]. São Paulo (SP): Faculdade de Saúde Pública, Universidade de São Paulo; 2002.

26. Hayashi F, Takimoto $\mathrm{H}$, Yoshital K, Yoshiikel N. Perceived body size and desire for thinness of young Japanese women: a population-based survey. $\mathrm{Br}$ J Nutr 2006; 96:1154-1162.

27. Schwartz MB, Brownell KD. Obesity and body image. Body Image 2004; 1:43-56.

28. Hill AJ, Williams J. Psychological health in a nonclinical sample of obese women. Int J Obes Relat M etab Disord 1998; 22(6):578-583.

29. Mack KA, Anderson L, Galuska D, Zablotsky D, Holtzman D, Ahluwalia I. Health and sociodemographic factors associated with body weight and weight objectives for women: 2000 behavioral risk factor surveillance system. J Womens Health 2004; 13(9):1019-1032. 
30. Bosi MLM, Luiz RR, Morgado CMC, Costa M LSC, Carvalho RJ. Autopercepção da imagem corporal entre estudantes de nutrição: um estudo no município do Rio de Janeiro. J Bras Psiquiatr 2006; 55(2):108-113.

Artigo apresentado em 01/02/2008

Aprovado em 08/05/2008 